\title{
Prediction of sudden cardiac death: next steps in pursuit of effective methodology
}

\author{
Eric C. Stecker • Sumeet S. Chugh
}

Received: 21 October 2010 /Accepted: 17 December 2010/Published online: 8 March 2011

(C) The Author(s) 2011. This article is published with open access at Springerlink.com

$\begin{array}{ll}\text { Abbreviations } \\ \text { CHF } & \text { Congestive heart failure } \\ \text { ECG } & \text { Electrocardiogram } \\ \text { HFNEF } & \text { Heart failure with normal ejection fraction } \\ \text { HRV } & \text { Heart rate variability } \\ \text { ICD } & \text { Implantable cardioverter defibrillator } \\ \text { LVEF } & \text { Left ventricular ejection fraction } \\ \text { MI } & \text { Myocardial infarction } \\ \text { NYHA } & \text { New York Heart Association } \\ \text { SAECG } & \text { Signal-averaged electrocardiogram } \\ \text { SCD } & \text { Sudden cardiac death } \\ \text { VT } & \text { Ventricular tachycardia }\end{array}$

The recognition that severe left ventricular dysfunction is a predictor of sudden cardiac death (SCD) risk enabled widespread utilization of the implantable cardioverter defibrillator (ICD) for SCD prevention. Use of the ICD prior to any signs or symptoms of ventricular arrhythmias ("primary prevention") is particularly important for SCD because case fatality for patients with sustained ventricular tachyarrhythmias is among the highest of any disease process. At the same time, primary prevention of SCD presents a unique epidemiologic challenge for many

E. C. Stecker

Cardiology Division, Oregon Health and Science University,

Portland, OR, USA

S. S. Chugh $(\bowtie)$

The Heart Institute, 5702 South Tower,

Cedars-Sinai Medical Center,

8700 Beverly Blvd,

Los Angeles, CA 90048, USA

e-mail: sumeet.chugh@cshs.org reasons [1, 2]: (1) high-risk subgroups constitute only a small proportion of all patients at risk for SCD, (2) the pathophysiologic etiologies of SCD are complex and infrequently recognized prior to arrest, (3) ventricular arrhythmia substrate often evolves over time and may require repeated risk stratification, (4) classification of arrhythmic death is particularly imprecise, (5) triggers for SCD may be transient, and (6) patients at high risk for SCD have many competing risks that are not ameliorated by ICDs. This review will address the limitations of risk stratification based on left ventricular ejection fraction (LVEF), summarize new developments in the field that extend beyond LVEF, and suggest new investigative approaches for refinement of SCD risk assessment.

\section{The promise of LVEF-based prevention efforts}

Two early trials, MADIT-I and Multicenter Unsustained Tachycardia Trial (MUSTT), established the role of ICDs for primary prevention of SCD based on impaired LVEF and positive electrophysiology study [3, 4]. Subsequent trials, Multicenter Automatic Defibrillator Implantation Trial II (MADIT-II) and Sudden Cardiac Death in Heart Failure Trial (SCD-HEFT), used LVEF without additional high-risk markers for ventricular arrhythmia. MADIT-II enrolled 1,232 patients with prior myocardial infarction (MI) and New York Heart Association (NYHA) class I, II, or III, and randomized to ICD versus conventional medical therapy [5]. SCD-HEFT enrolled 2,521 patients with ischemic or nonischemic cardiomyopathy and NYHA class II or III congestive heart failure (CHF) and randomized to ICD, amiodarone, or placebo [6]. MADIT-II survival curves showed a $31 \%$ reduction in risk of death $(5.6 \%$ absolute risk reduction at 20-month mean follow-up), while SCD-HEFT showed a $23 \%$ 
reduction in risk of death in ICD as compared to placebo (7.2\% absolute risk reduction at 5-year follow-up). There was no benefit of amiodarone as compared to placebo.

\section{Limitations of LVEF-based prevention efforts}

It quickly became evident that a significant number of patients who receive an ICD under MADIT-II or SCD-HEFT criteria do not benefit from it during intermediate-term follow-up of 25 years [7]. During the 20-month average follow-up of MADIT-II, the annualized rate of appropriate ICD therapies was $17 \%$ [8]. Over 5 years of follow-up in SCD-HEFT, the annualized rate of appropriate therapies was 5.1\% [6]. Differences in patient populations likely were notable contributors to the disparate rates of ICD utilization. Consistent between both studies was a lack of benefit observed at the upper range of the LVEF limit for enrollment [1]. At the same time that many ICDs go unutilized, a majority of patients at risk for cardiac arrest do not qualify for an ICD despite the broadened LVEF-based indications (conceptually illustrated in Fig. 1 and in Table 1). Two population based studies in the Netherlands (Maastricht study) and Oregon, USA (The Oregon Sudden Unexpected Death Study), have demonstrated that the majority of patients who suffer SCD do not have a severely reduced LVEF. In subsets with medical history available, only $20-30 \%$ of patients who suffered SCD had LVEF low enough to have warranted primary prevention of SCD with an ICD [9-11].

Two conclusions can be drawn about current practices for primary prevention ICD use:

1. Even at the higher $17 \%$ annualized utilization rates from MADIT-II, there is considerable room for improvement in patient selection if new risk stratification methods can be developed.
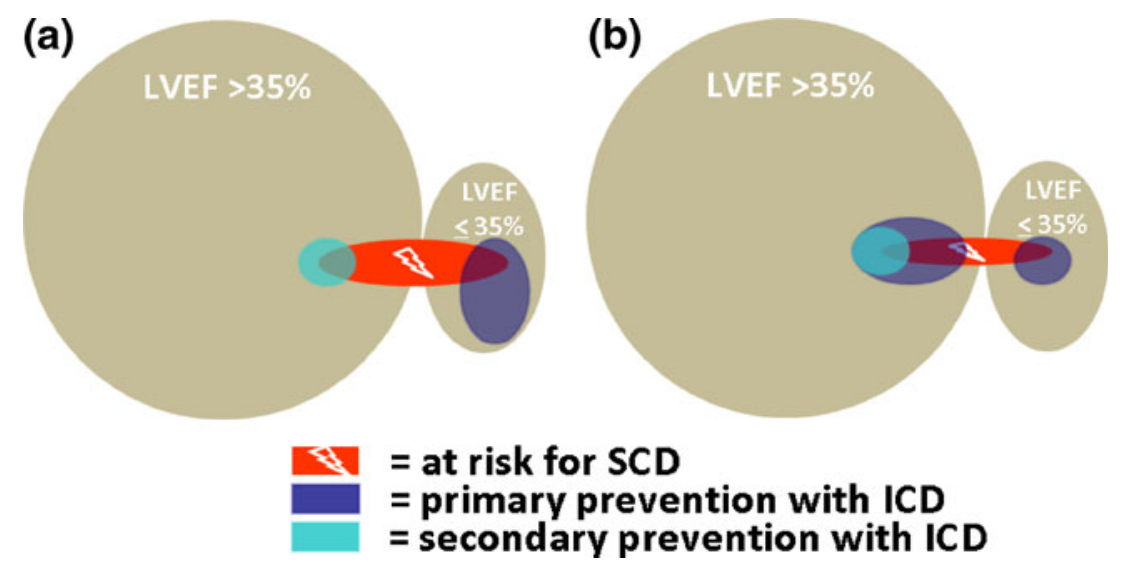

\section{Limitations of existing non-LVEF risk predictors}

A number of non-LVEF risk stratification tests have been evaluated individually for relevance in predicting a variety of outcomes (overall mortality, arrhythmic events/mortality, ICD shocks, and mortality benefit from ICDs). These tests include signal-averaged ECG, QRS duration, QT interval, ventricular ectopy, heart rate variability, heart rate turbulence, exercise capacity, heart failure class, heart rate recovery or ventricular ectopy after exercise, T-wave alternans, baroreceptor sensitivity, and electrophysiology study with ventricular programmed ventricular stimulation (many summarized in Table 2) [12, 13]. Clinical applicability has been limited thus far because most studies have not demonstrated sufficiently high predictive value for arrhythmic death or arrhythmic events [12]. Five were considered promising enough to be tested for their ability to predict mortality benefit from ICD use in the context of randomized trials. The first two, nonsustained ventricular tachycardia (VT) on ambulatory ECG and inducible VT during electrophysiology study, were effective in defining patients with severe ischemic cardiomyopathy who are at very high risk of SCD and benefit from an ICD [3, 4]; however, the SCD risk among patients without these markers was still uncomfortably high for patients with severely reduced LVEF, prompting subsequent trials without these predictors $[5,6]$. The third non-LVEF risk stratifier, abnormal signal-averaged ECG (SAECG), was

Fig. 1 Venn diagrams of primary prevention and secondary prevention ICD utilization strategies, (a) shows the current approach, with a majority of SCD victims not previously qualifying for an ICD and many ICDs unutilized, and (b) shows an idealized approach with

fewer cases of SCD due to fewer at-risk patients (from coronary artery disease prevention efforts) and more efficient utilization of ICDs among at-risk patients. $L V E F$ left ventricular ejection fraction, $S C D$ sudden cardiac arrest, ICD implantable cardioverter defibrillator 
Table 1 Estimates of SCD incidence in Portland, Oregon metro area population (aged $\geq 45$ years)

\begin{tabular}{llll}
\hline Ejection fraction & Percent of population & Percent of SCDs & SCD incidence rate \\
\hline All & $100 \%$ & $100 \%$ & $142 / 100,000$ \\
LVEF $\leq 50 \%$ & $5.8 \%$ & $52 \%$ & Greater than $1,000 / 100,000$ \\
LVEF $>50 \%$ & $94.2 \%$ & $48 \%$ & Less than $100 / 100,000$ \\
\hline
\end{tabular}

The SCD incidence among patients with normal LVEF is very small, yet accounts for almost half of all SCD cases. This illustrates that in order to significantly impact the overall problem of SCD, prevention efforts must focus beyond LVEF. Estimates in this table should be used for general descriptive purposes only. We generated estimates of SCD incidence rates for normal versus abnormal LVEF by using results from the populationbased Rochester Epidemiology Project [35] and applying to the same age distribution for the population-based Oregon Sudden Unexpected Death Study [10]. We assumed that the LVEF distribution for SCD patients with prior LVEF assessment applied to all patients with SCD [11]. Because of these extrapolations, we have reported ranges of SCD incidence for LVEF subcategories rather than the calculated SCD point incidence because such an incidence would have precision highly disproportionate to its level of accuracy. $L V E F$ left ventricular ejection fraction, $S C D$ sudden cardiac arrest, ICD implantable cardioverter defibrillator

used as an entry criterion in the CABG-PATCH trial [14]. Although SAECG had shown initial promise, there was no benefit from ICD implantation in this trial. The fourth predictor, heart rate variability on 24-h Holter, was tested as an entry criterion for the Defibrillator in Acute Myocardial Infarction Trial (DINAMIT) of ICD placement within 6 to 40 days of MI in patients with LVEF $\leq 35 \%$ [15]. This trial also showed no benefit. The fifth predictor, microvolt $\mathrm{T}$ wave alternans, has shown some promise, but when tested in the setting of primary prevention ICD use as part of SCDHeFT, it was not predictive of arrhythmic events [16].

\section{Identification of novel non-LVEF risk predictors}

Novel risk prediction methods are acutely needed to more efficiently utilize ICDs for SCD prevention. We currently spend approximately $\$ 3.6$ billion dollars annually on ICDs in the US (113,000 annual implants [17] with an assumed cost per implant of $\$ 33,000$ [18]) with a large majority of devices unutilized. At the same time, current ICD guidelines do not address half of patients who will experience SCD [11]. We believe effective and efficient SCD prevention will require two simultaneous efforts:

\subsection{Refine existing criteria for ICD-based primary prevention using clinical risk scores}

Risk scores have been used to retrospectively define benefit or lack of benefit from primary prevention ICD use. Investigators from the MUSTT study identified EFindependent predictors of total and arrhythmic mortality among patients with LVEF $\leq 40 \%$ after MI who were assigned to the no-antiarrhythmic drug/no-ICD arm [19]. The MADIT-II investigators derived a risk score based on patient data from MADIT-II and compared the mortality rates for ICD or conventional therapy at different risk-score levels [20]. Very low and very high mortality rate cohorts did not benefit from ICDs, while intermediate risk cohorts had a $49 \%$ risk reduction. SCD-HeFT patients were similarly evaluated using a risk score modified from the Seattle Heart Failure Model (SHFM-D) and derived in a separate population [21]. Investigators evaluated ICD benefit in each mortality quintile. Mortality risk reductions for ICD therapy ranged from $31 \%$ to $54 \%$ in first four quintiles, with no benefit in the highest risk quintile. The absolute risk reductions were $7 \%$ to $14 \%$ in the first four quintiles over nearly 4 years follow-up.

The MADIT-II and SHFM-D risk scores use non-invasive data regarding co-mordibities, functional status, and laboratory values that could be easily utilized in clinical practice. The MADIT-II score applies only to ischemic cardiomyopathy patients, while the SHFM-D score from SCD-HeFT applies to ischemic and non-ischemic cardiomyopathy patients. The SHFM-D score has 15 predictor variables (including LVEF) while the MADIT-2 score has five predictor variables and was derived separately from the SCD-HeFT validation population. Both studies show that patients with the highest overall mortality rates did not benefit from ICDs.

It may be difficult for some physicians and patients to move toward withholding ICDs based on these risk scores because they were not used as entry criteria for randomized controlled trials. The uncertainty would be clarified by a randomized trial designed to test non-inferiority of a strategy to withhold ICD treatment based on risk score for specific patients with LVEF $\leq 35 \%$. While there are challenges to the conduct of such a trial, the cost of such a reseach effort would be a more fraction of the current total annual ICD expenditures in the US. Even if this research effort cost $\$ 100$ million, it would represent only 3\% of total annual ICD expenditures in the US.

\subsection{Define new high-risk, ICD-eligible patients}

Develop new risk stratification tools from large unbiased community-based evaluations where all-comers with SCD are evaluated regardless of LVEF Additional novel risk 
Table 2 Summary of existing non-LVEF risk stratification tests

\begin{tabular}{|c|c|c|}
\hline Risk stratification tests & Evidence for predicting SCD & Evidence for predicting ICD-detected VT/VF \\
\hline $\begin{array}{l}\text { Programmed ventricular } \\
\text { stimulation }\end{array}$ & $\begin{array}{l}\text { - Strong PPV in ischemic cardiomyopathy; also } \\
\text { proven to identify patients with large mortality } \\
\text { benefit from ICDs. } \\
\text { - Limited negative predictive value in ischemic } \\
\text { cardiomyopathy with moderately to severely } \\
\text { reduced LVEF. } \\
\text { - Not predictive for other cardiomyopathy } \\
\text { etiologies. }\end{array}$ & $\begin{array}{l}\text { Strong PPV for ventricular arrhythmia risk in ischemic } \\
\text { cardiomyopathy. }\end{array}$ \\
\hline QRS duration & $\begin{array}{l}\text { Mixed findings, MUSTT found it was } \\
\text { independently predictive while other studies } \\
\text { did not. }\end{array}$ & $\begin{array}{l}\text { Preponderance of studies shows it is not independently } \\
\text { predictive. }\end{array}$ \\
\hline QT interval & $\begin{array}{l}\text { Population-based and cohort data showing } \\
\text { independent prediction of SCD. }\end{array}$ & \\
\hline T-wave alternans & \multicolumn{2}{|c|}{$\begin{array}{l}\text { Potentially promising technique, but several large cohort studies have shown limited PPV and not predictive in a large } \\
\text { randomized trial. Generally composite outcomes measured. }\end{array}$} \\
\hline Signal-averaged ECG & $\begin{array}{l}\text { Limited to post-MI patients. Variable PPV and no } \\
\text { benefit for ICD implantation among positive test in } \\
\text { CABG-PATCH. Negative predictive value has } \\
\text { been }>90 \% \text {, though generally evaluated prior to } \\
\text { widespread reperfusion of AMI. }\end{array}$ & \\
\hline $\begin{array}{l}\text { Heart rate variability } \\
\text { and baroreflex } \\
\text { sensitivity }\end{array}$ & $\begin{array}{l}\text { Limited to post-MI patients. Aside from ATRAMI, } \\
\text { these markers have not discriminated sudden from } \\
\text { non-sudden death. }\end{array}$ & \\
\hline $\begin{array}{l}\text { Cardiac MRI with late } \\
\text { gadolinium } \\
\text { enhancement imaging }\end{array}$ & & $\begin{array}{l}\text { Correlates with surrogate markers of SCD risk in patients } \\
\text { with ischemic, non-ischemic, and hypertrophic cardiomy- } \\
\text { opathies in small studies }(n<200 \text { for each study). }\end{array}$ \\
\hline
\end{tabular}

Several tests also predict all-cause mortality and/or cardiac events and mortality; only tests with purported or demonstrated preferential discrimination for arrhythmic events and sudden cardiac death are addressed here. See also more detailed discussions of risk stratification tests $[12,13] . L V E F$ left ventricular ejection fraction, $S C D$ sudden cardiac arrest, $I C D$ implantable cardioverter defibrillator, $M I$ myocardial infarction, $M R I$ magnetic resonance imaging, $P P V$ positive predictive value

factors (clinical characteristics, biomarkers, genetic variation, etc.) could be identified from large community-based studies that collect SCD patients who have a broad range of LVEF. For example, genome-wide association studies performed in the community can yield novel genetic variations that confer protectiveness [22] as well as susceptibility [23] to SCD. Biomarkers such as C-reactive protein, B-natriuretic peptide, and fatty acid levels have been associated with SCD risk [22]. Cardiac imaging techniques have also shown promise. Nuclear imaging of cardiac sympathetic nerve activity and perfusion may identify a higher arrhythmia-risk substrate [24, 25]. Cardiac magnetic resonance imaging (MRI) showing late gadolinium enhancement (presence of scar) in patients with ischemic cardiomyopathy appears highly predictive of inducibility of sustained VT with programmed electrical stimulation [26]. Among patients with non-ischemic cardiomyopathy, the presence of late gadolinium enhancement on cardiac MRI is predictive of a composite endpoint including ICD discharges [27]. Studies of patients with hypertrophic cardiomyopathy have shown correlation between presence of LGE on cardiac MRI and markers of SCD risk or composite endpoints including SCD [28-30]. New techniques for evaluating interstitial fibrosis by MRI are undergoing development and evaluation.

Because many genetic and clinical variables have odds ratios or relative risk ratios of 2 or less, it will be important to derive multiple independent variables from the same population. Ultimately, these factors should be combined into cost-efficient test panels.

Utilize existing and novel risk stratification methods to identify patient populations with LVEF $>35 \%$ whose annual SCD rates are at least 5\% Identifying groups with annual SCD rates greater than $5 \%$ would identify patients at higher risk than existing primary prevention ICD trials. This would increase odds of efficient of ICD utilization in subsequent trials. Risk scores using clinical factors, biomarkers, and genetic factors can be tested retrospectively in some large community-based cohorts or drug trial cohorts when modes of death are well characterized and blood/serum has been stored for subsequent analysis. Other risk stratification tests will generally require evaluation prospectively (e.g., imaging studies, novel electrocardio- 
graphic methods, etc.). After identifying new patient groups with high annual SCD rates, we must evaluate the ability of ICDs to reduce all-cause mortality.

\section{Translation of novel strategies into clinical practice}

There are several pathophysiologically homogenous cohorts with LVEF $>35 \%$ that may be sufficiently high risk to warrant randomized controlled trials of primary prevention ICD use:

- Acute MI. Within the first 30 days after MI, $1.4 \%$ of patients have SCD (annualized rate of 17\% [31]). Two randomized controlled trials, DINAMIT and Immediate Risk-Stratification Improves Survival (IRIS), tested the value of ICDs in patients in the first 40 days after MI and showed no benefit $[15,32]$. It is not clear whether these negative trials resulted from excessive risk of nonarrhythmic death during this time period, ventricular arrhythmia that is less amenable to ICD termination (due to recurrent acute infarction or pump failure), or risk stratification methods that did not select patients at sufficient risk for SCD. A large majority of patients in DINAMIT and IRIS were selected based on impaired LVEF combined with either impaired heart rate variability or elevated resting heart rate, both of which are poor predictors of arrhythmic events. Alternative risk stratification methods such as electrophysiology study [33] and/or MRI [34] could yield patients with higher arrhythmic event rates.

- Ischemic CM with LVEF $>35 \%$. Beyond the first month after MI, the SCD rate drops rapidly and is lower for patients with higher initial LVEF [31]. Nonetheless, SCD rates for those with LVEF $>35 \%$ in the first year after MI remain at approximately 3\% (equivalent to placebo annual arrhythmic death rate in SCD-HeFT). With appropriate risk stratification methods (for example, a combination of class $3 \mathrm{CHF}$, fragmented QRS, electrophysiology study, MRI, etc.), it is quite plausible to identify a population at higher SCD risk compared to existing primary prevention ICD patients.

- Heart failure with normal ejection fraction (HFNEF). Approximately $50 \%$ of patients with congestive heart failure have normal or near-normal LVEF [35]. The proportion of deaths due to ventricular arrhythmia in this group is likely significantly less than in heart failure with impaired LVEF. In fact, in the Duke database, the rate of SCD was $0.5 \%$ per year for patients with HFNEF [36]. However, several independent predictors of SCD were identified which, together with screening biomarkers or genetic studies, could identify patients at sufficient risk for ICD-based primary prevention. This group is important to target since one half of all SCDs occur in patients with normal LVEF $[9,11]$.

- Non-ischemic cardiomyopathy LVEF $>35 \%$. Although sudden death rates are too low to warrant ICD prevention in this overall group, there may be subpopulations at sufficiently high risk to benefit from primary prevention ICD use. Risk stratification methods are not well developed, but this group will be important to target for prevention for the estimated $20 \%$ or so of patients who have SCD without significant coronary artery disease [22].

Regardless of high arrhythmic event or SCD rates in observational studies, randomized clinical trials will be necessary to prove ICD benefit in individual population subgroups. While measuring arrhythmic events increases the power of an observational study, SCD rates are substantially lower than arrhythmic event rates due to the often non-sustained nature of ventricular arrhythmias. Even high observed SCD rates do not ensure benefit from ICDs. Improvements in SCD rates can be completely offset by increases in competing causes of mortality [15, 32, 37]. To the degree that competing mortality is driven by progressive heart failure/pump failure, use of ICDs with biventricular pacing capability may further improve ICD efficacy. The economic viability of any prevention strategy using ICDs will be dependent not only on magnitude of mortality benefit, but also cost of the ICD systems used. In general, physicians and medical device manufacturers have favored the use of ICDs with maximal functionality (and higher costs). Clinical trials utilizing less expensive devices in lower risk patient populations could expand SCD prevention. However, any such strategy would be subject to physician, patient (and sometimes legal) bias toward high technology-high cost medicine.

\section{Conclusions}

Left ventricular systolic dysfunction has served for many years as an important tool for SCD risk identification and subsequent prevention efforts. While it continues to benefit a subgroup of patients at risk of $\mathrm{SCD}$, the predictive ability of this test among overall patients at risk for SCD has been overestimated. A continued, aggressive search for novel predictors other than LVEF is warranted using a pathophysiologically targeted approach. Development of tests to refine SCD risk stratification and the conduct of trials to translate these to clinical practice will require more ongoing effort and expense. However, in the long run, these ongoing efforts are the answer to optimizing the utilization of an important preventive intervention such as the ICD, and preventing premature death due to SCD. 
Acknowledgement Funded by National Heart Lung and Blood Institute R01HL088416, R01 HL088416-03 S1 and R01 HL10517001. Dr. Chugh is the Pauline and Harold Price Professor of Cardiac Electrophysiology at the Cedars-Sinai Heart Institute, Los Angeles, CA. We are indebted to Dr. Kyndaron Reinier for her critical review of this manuscript.

Disclosures Dr. Stecker has licensed biventricular pacing lead technology to Medtronic; he does not receive ongoing royalties. Dr. Chugh has no relationships to disclose.

Open Access This article is distributed under the terms of the Creative Commons Attribution Noncommercial License which permits any noncommercial use, distribution, and reproduction in any medium, provided the original author(s) and source are credited.

\section{References}

1. Myerburg, R. J., Reddy, V., \& Castellanos, A. (2009). Indications for implantable cardioverter-defibrillators based on evidence and judgment. Journal of the American College of Cardiology, 54, 747-763.

2. Zipes, D. P., Camm, A. J., Borggrefe, M., et al. (2006). ACC/ AHA/ESC 2006 guidelines for management of patients with ventricular arrhythmias and the prevention of sudden cardiac death: a report of the American College of Cardiology/American Heart Association Task Force and the European Society of Cardiology Committee for Practice Guidelines (Writing Committee to Develop Guidelines for Management of Patients with Ventricular Arrhythmias and the Prevention of Sudden Cardiac Death): developed in collaboration with the European Heart Rhythm Association and the Heart Rhythm Society. Circulation, 114, e385-e484.

3. Moss, A. J., Hall, W. J., Cannom, D. S., et al. (1996). Improved survival with an implanted defibrillator in patients with coronary disease at high risk for ventricular arrhythmia. The New England Journal of Medicine, 335, 1933-1940.

4. Buxton, A. E., Lee, K. L., Fisher, J. D., et al. (1999). A randomized study of the prevention of sudden death in patients with coronary artery disease. Multicenter Unsustained Tachycardia Trial Investigators. The New England Journal of Medicine, 341, 1882-1890.

5. Moss, A. J., Zareba, W., Hall, W. J., et al. (2002). Prophylactic implantation of a defibrillator in patients with myocardial infarction and reduced ejection fraction. The New England Journal of Medicine, 346, 877-883.

6. Bardy, G. H., Lee, K. L., Mark, D. B., et al. (2005). Amiodarone or an implantable cardioverter-defibrillator for congestive heart failure. The New England Journal of Medicine, 352, 225-237.

7. Goldenberg, I., \& Moss, A. J. (2008). Implantable device therapy. Progress in Cardiovascular Diseases, 50, 449-474.

8. Singh, J. P., Hall, W. J., McNitt, S., et al. (2005). Factors influencing appropriate firing of the implanted defibrillator for ventricular tachycardia/fibrillation: findings from the Multicenter Automatic Defibrillator Implantation Trial II (MADIT-II). Journal of the American College of Cardiology, 46, 1712-1720.

9. Gorgels, A. P., Gijsbers, C., de Vreede-Swagemakers, J., et al. (2003). Out-of-hospital cardiac arrest-the relevance of heart failure. The Maastricht Circulatory Arrest Registry. European Heart Journal, 24, 1204-1209.

10. Chugh, S. S., Jui, J., Gunson, K., et al. (2004). Current burden of sudden cardiac death: multiple source surveillance versus retro- spective death certificate-based review in a large U.S. community. Journal of the American College of Cardiology, 44, 1268-1275.

11. Stecker, E. C., Vickers, C., Waltz, J., et al. (2006). Populationbased analysis of sudden cardiac death with and without left ventricular systolic dysfunction: two-year findings from the Oregon Sudden Unexpected Death Study. Journal of the American College of Cardiology, 47, 1161-1166.

12. Buxton, A. E. (2009). Risk stratification for sudden death in patients with coronary artery disease. Heart Rhythm, 6, 836-847.

13. Goldberger, J. J., Cain, M. E., Hohnloser, S. H., et al. (2008). American Heart Association/American College of Cardiology Foundation/Heart Rhythm Society scientific statement on noninvasive risk stratification techniques for identifying patients at risk for sudden cardiac death: a scientific statement from the American Heart Association Council on Clinical Cardiology Committee on Electrocardiography and Arrhythmias and Council on Epidemiology and Prevention. Journal of the American College of Cardiology, 52, 1179-1199.

14. Bigger, J. T., Jr. (1997). Prophylactic use of implanted cardiac defibrillators in patients at high risk for ventricular arrhythmias after coronary-artery bypass graft surgery. Coronary Artery Bypass Graft (CABG) Patch Trial Investigators. The New England Journal of Medicine, 337, 1569-1575.

15. Hohnloser, S. H., Kuck, K. H., Dorian, P., et al. (2004). Prophylactic use of an implantable cardioverter-defibrillator after acute myocardial infarction. The New England Journal of Medicine, 351, 2481-2488.

16. Gold, M. R., Ip, J. H., Costantini, O., et al. (2008). Role of microvolt T-wave alternans in assessment of arrhythmia vulnerability among patients with heart failure and systolic dysfunction: primary results from the T-wave alternans sudden cardiac death in heart failure trial substudy. Circulation, 118, 2022-2028.

17. Hammill, S. C., Kremers, M. S., Kadish, A. H., et al. (2009). Review of the ICD Registry's third year, expansion to include lead data and pediatric ICD procedures, and role for measuring performance. Heart Rhythm, 6, 1397-1401.

18. Zwanziger, J., Hall, W. J., Dick, A. W., et al. (2006). The cost effectiveness of implantable cardioverter-defibrillators: results from the Multicenter Automatic Defibrillator Implantation Trial (MADIT)-II. Journal of the American College of Cardiology, 47, 2310-2318.

19. Buxton, A. E., Lee, K. L., Hafley, G. E., et al. (2007). Limitations of ejection fraction for prediction of sudden death risk in patients with coronary artery disease: lessons from the MUSTT study. Journal of the American College of Cardiology, 50, 1150-1157.

20. Goldenberg, I., Vyas, A. K., Hall, W. J., et al. (2008). Risk stratification for primary implantation of a cardioverterdefibrillator in patients with ischemic left ventricular dysfunction. Journal of the American College of Cardiology, 51, 288-296.

21. Levy, W. C., Lee, K. L., Hellkamp, A. S., et al. (2009). Maximizing survival benefit with primary prevention implantable cardioverter-defibrillator therapy in a heart failure population. Circulation, 120, 835-842.

22. Chugh, S. S. (2010). Early identification of risk factors for sudden cardiac death. Nature reviews, 7, 318-326.

23. Bezzina, C. R., Pazoki, R., Bardai, A., et al. (2010). Genome-wide association study identifies a susceptibility locus at $21 \mathrm{q} 21$ for ventricular fibrillation in acute myocardial infarction. Nature Genetics, 42, 688-691.

24. Kioka, H., Yamada, T., Mine, T., et al. (2007). Prediction of sudden death in patients with mild-to-moderate chronic heart failure by using cardiac iodine-123 metaiodobenzylguanidine imaging. Heart, 93, 1213-1218.

25. Nishisato, K., Hashimoto, A., Nakata, T., et al. (2010). Impaired cardiac sympathetic innervation and myocardial perfusion are related to lethal arrhythmia: quantification of cardiac tracers in patients with ICDs. Journal of Nuclear Medicine, 51, 1241-1249. 
26. Schmidt, A., Azevedo, C. F., Cheng, A., et al. (2007). Infarct tissue heterogeneity by magnetic resonance imaging identifies enhanced cardiac arrhythmia susceptibility in patients with left ventricular dysfunction. Circulation, 115, 2006-2014.

27. Wu, K. C., Weiss, R. G., Thiemann, D. R., et al. (2008). Late gadolinium enhancement by cardiovascular magnetic resonance heralds an adverse prognosis in nonischemic cardiomyopathy. Journal of the American College of Cardiology, 51, 2414-2421.

28. Adabag, A. S., Maron, B. J., Appelbaum, E., et al. (2008). Occurrence and frequency of arrhythmias in hypertrophic cardiomyopathy in relation to delayed enhancement on cardiovascular magnetic resonance. Journal of the American College of Cardiology, 51, 1369-1374.

29. Moon, J. C., Mogensen, J., Elliott, P. M., et al. (2005). Myocardial late gadolinium enhancement cardiovascular magnetic resonance in hypertrophic cardiomyopathy caused by mutations in troponin I. Heart, 91, 1036-1040.

30. O'Hanlon, R., Grasso, A., Roughton, M., et al. (2010). Prognostic significance of myocardial fibrosis in hypertrophic cardiomyopathy. Journal of the American College of Cardiology, 56, 867-874.

31. Solomon, S. D., Zelenkofske, S., McMurray, J. J., et al. (2005). Sudden death in patients with myocardial infarction and left ventricular dysfunction, heart failure, or both. The New England Journal of Medicine, 352, 2581-2588.
32. Steinbeck, G., Andresen, D., Seidl, K., et al. (2009). Defibrillator implantation early after myocardial infarction. The New England Journal of Medicine, 361, 1427-1436.

33. Zaman, S., Sivagangabalan, G., Narayan, A., et al. (2009). Outcomes of early risk stratification and targeted implantable cardioverter-defibrillator implantation after ST-elevation myocardial infarction treated with primary percutaneous coronary intervention. Circulation, 120, 194-200.

34. de Waha, S., Desch, S., Eitel, I., et al. (2010). Impact of early vs. late microvascular obstruction assessed by magnetic resonance imaging on long-term outcome after ST-elevation myocardial infarction: a comparison with traditional prognostic markers. European Heart Journal, 31(21), 2660-2668.

35. Redfield, M. M., Jacobsen, S. J., Burnett, J. C., Jr., et al. (2003). Burden of systolic and diastolic ventricular dysfunction in the community: appreciating the scope of the heart failure epidemic. JAMA, 289, 194-202.

36. Al-Khatib, S. M., Shaw, L. K., O'Connor, C., et al. (2007). Incidence and predictors of sudden cardiac death in patients with diastolic heart failure. Journal of Cardiovascular Electrophysiology, 18, 1231-1235.

37. Lee, D. S., Tu, J. V., Austin, P. C., et al. (2007). Effect of cardiac and noncardiac conditions on survival after defibrillator implantation. Journal of the American College of Cardiology, 49, 2408-2415. 